\title{
Adenoviral Vectors Armed with PAPILLOMAVIRUs Oncogene Specific CRISPR/Cas9 Kill Human-Papillomavirus-Induced Cervical Cancer Cells
}

\author{
Eric Ehrke-Schulz, Sonja Heinemann, Lukas Schulte, Maren Schiwon and Anja Ehrhardt * \\ Institute for Virology and Microbiology, Department for Human Medicine, Faculty of Health, \\ Center for Biomedical Education and Research (ZBAF), Witten/Herdecke University, Stockumer Street 10, \\ 58453 Witten, Germany; Eric.Ehrke-Schulz@uni-wh.de (E.E.-S.); Sonja.Heinemann@uni-wh.de (S.H.); \\ Lukas.Schulte@uni-wh.de (L.S.); m.schiwon@gmx.de (M.S.) \\ * Correspondence: anja.ehrhardt@uni-wh.de
}

Received: 15 May 2020; Accepted: 14 July 2020; Published: 17 July 2020

\begin{abstract}
Human papillomaviruses (HPV) cause malignant epithelial cancers including cervical carcinoma, non-melanoma skin and head and neck cancer. They drive tumor development through the expression of their oncoproteins E6 and E7. Designer nucleases were shown to be efficient to specifically destroy HPV16 and HPV18 oncogenes to induce cell cycle arrest and apoptosis. Here, we used high-capacity adenoviral vectors (HCAdVs) expressing the complete CRISPR/Cas9 machinery specific for HPV18-E6 or HPV16-E6. Cervical cancer cell lines SiHa and CaSki containing HPV16 and HeLa cells containing HPV18 genomes integrated into the cellular genome, as well as HPV-negative cancer cells were transduced with HPV-type-specific CRISPR-HCAdV. Upon adenoviral delivery, the expression of HPV-type-specific CRISPR/Cas9 resulted in decreased cell viability of HPV-positive cervical cancer cell lines, whereas HPV-negative cells were unaffected. Transduced cervical cancer cells showed increased apoptosis induction and decreased proliferation compared to untreated or HPV negative control cells. This suggests that HCAdV can serve as HPV-specific cancer gene therapeutic agents when armed with HPV-type-specific CRISPR/Cas9. Based on the versatility of the CRISPR/Cas9 system, we anticipate that our approach can contribute to personalized treatment options specific for the respective HPV type present in each individual tumor.
\end{abstract}

Keywords: papillomavirus; HPV; CRISPR; gene therapy; viral vector; adenovirus

\section{Introduction}

Human papillomaviruses (HPV) are small non-enveloped epitheliotropic DNA viruses with a circular genome comprised of approximately 8000 base pairs (bp). So called high-risk HPV are responsible for the development of malignant cervical carcinoma and their precursor lesions cervical intraepithelial neoplasia (CIN) [1]. Currently over 200 different HPV types are known. Due to their carcinogenic properties, especially HPV16, 18 and 31 (but also 33, 35, 39, 45, 51, 52, 56, 58, 59, 68, 73, and 82) are classified as high-risk HPV types [2]. HPV DNA was also found in oropharyngeal carcinomas summarized as head and neck cancer (HNC) and are therefore regarded as important carcinogens [3]. Furthermore, HPV are a cofactor in the development of dermatologic malignancies, such as Squamous cell carcinoma (SCC) and basal cell carcinoma (BCC) of the skin summarized as non-melanoma skin cancer (NMSC), and their precursor lesions, actinic keratosis (AK) [4]. Despite the availability of a protective vaccination against high-risk HPV, not all girls and young women (as well as boys and young men) are vaccinated. Therefore, it is likely, that HPV-associated tumors represent 
a continuous health care burden. HPV-associated tumors such as cervical carcinoma, NMSC and HNC can be removed surgically, but a complete detection and removal of tumor tissue including possible precursor lesions in the immediate vicinity is difficult as non-infected regions, HPV infected but non transformed regions, precursor lesions and invasive carcinomas are located in close proximity of an epithelial area [5]. The conventional cancer therapies such as radiation or chemotherapy in concert with surgical resection increase the chances of recovery but are associated with strong side effects. Therefore, the development and testing of alternative treatment strategies are desirable.

In the context of cervical infection, the HPV genome can be integrated into the chromosomal DNA of basal epithelial cells. During this process, the episomal, circular HPV genome is linearized within the HPV E2 gene, leading to a loss of E2 function. As the HPV E2 protein negatively regulates HPV E6 and E7 gene expression, the loss of HPV E2 function leads to unregulated overexpression of the HPV oncogenes E6 and E7, that mediate transformation of the host cell by inducing cell proliferation, bypassing cell cycle control and inhibition of apoptosis. The HPV E6 protein interacts with the tumor suppressor protein p53, causes its degradation and thus prevents cell cycle control and the initiation of apoptosis [6]. The HPV E7 protein interacts with the retinoblastoma protein pRB and causes the release of the pRB-bound E2F transcription factor. E2F subsequently induces the expression of genes that induce cell cycle progression $[7,8]$. In recent years, several approaches have been described to induce cell cycle arrest and induction of apoptosis in HPV positive cancer cells, either by specifically inhibiting HPV oncoprotein interactions using intracellular antibodies [9] or peptides such as HPV E7 antagonist [10], E6-binding aptamer [11], E6-AP mimetic epitope [12], or RNA molecules like Anti-E6 ribozyme [13]. Moreover, downregulating HPV oncogene expression using RNA interference [14-21] has been shown to be a promising treatment option for HPV induced tumors. However, only the most recent studies tried to translate these in vitro findings toward in vivo applications using non-viral RNA delivery or AAV-mediated viral delivery.

Designer nucleases such as zinc finger nucleases (ZFN), transcription activator-like effector nuclease (TALEN) and especially clustered regularly interspaced short palindromic repeats (CRISPR/Cas9) are highly efficient customizable molecular scissors for sequence-specific induction of in/del mutations at the DNA-target site. This gene disruption strategy has been applied in antiviral approaches against DNA viruses such as Hepatis B virus (HBV) [22-24]. In the context of HPV-related cancer, it was reported that cleavage of the HPV18 origin of replication by artificial zinc-finger proteins fused to a bacterial nuclease inhibited HPV DNA replication [25]. CRISPR/Cas9 mediated inactivation of HPV18- and HPV16 E6 or E7 resulted in the induction of p53 or pRb, leading to cell cycle arrest and cell death [26]. CRISPR/Cas9 mediated disruption of HPV E7 open reading frame (orf) alone induced apoptosis and growth inhibition in HPV16 positive cervical cancer cells [27] and HPV6/11 E7-expressing keratinocytes [28]. Moreover, it was shown that a CRISPR/Cas9 approach to specifically destroy the HPV16 early promoter, HPV16 E6, or HPV16 E7 coding regions lead to decreased cell viability and increased apoptosis induction and decreased growth of SiHa cell-derived tumors implants in BALB/C nude mice [29]. Interestingly cleavage of HPV16 E6 alone was sufficient to induce apoptosis and growth inhibition of HPV16-positive cells [30].

However, delivery approaches to enable comprehensive in preclinical in vivo studies are rare. A recent study showed that non-viral CRISPR/Cas9 delivery using PEGylated liposomes resulted in tumor elimination in vivo [31]. Numerous publications reported the CRISPR/Cas9 delivery using AAV co-transduction approaches. However, these vectors are rather small and do not allow to deliver all CRISPR/Cas9 components including one or several guide RNAs within one vector. Even though CRISPR/Cas9 is particularly suitable for arming conditionally replicating Adenoviruses (CRAdVs)/oncolytic Adenoviruses [32-34], the potential of viral delivery of HPV-specific CRISPR/Cas9 was not fully exploited. Adenoviruses (AdV) infect a great variety of different cells types and tissues and can enter quiescent as well as dividing cells. Another benefit of the AdV vector system arises from the non-integrating, episomal persistence of the viral genome [35]. As AdV do not integrate their genome into host cell chromosomes genotoxicity related to insertion into transcribed genomic 
loci is circumvented. To translate the non-viral CRISPR approaches of the preceding studies into an all-in-one viral vector delivery approach to enable future in vivo studies (high-capacity adenoviral vectors $(\mathrm{HCAdV})$ ) offer several benefits. In HCAdV genomes all viral coding sequences have been removed and only the inverted terminal repeats (ITR) and the packaging signal that is necessary for vector genome replication and efficient genome packaging are still present [36,37]. The HCAdV packaging capacity of up to $35 \mathrm{~kb}$ allows transporting the whole CRISPR/Cas9 machinery including several gRNAs [38]. As HCAdV do not express AdV genes, they are regarded as less immunogenic than early generation AdV vectors $[39,40]$. Nevertheless, production of HCAdV are time and work intensive when compared with Lentivirus- or AAV-vector platforms, hampering their exploration for specific applications.

In this study, we aim to exploit the advantages of the HCAdV platform and constructed HCAdVs expressing the complete CRISPR/Cas9 machinery including Cas9 and a gRNA specific for HPV16-E6 [29] or HPV18E6 [26,38] within a single vector. These non-replicating HCAdV vectors express the HPV E6 specific CRISPR/Cas9 and destroy the respective E6 oncogene. Upon vector transduction of HPV positive cervical carcinoma cell lines HeLa, CaSki, and SiHa and HPV-negative lung carcinoma cell line A549, we examined the potential of the HPV E6 specific CRISPR/Cas9 expressing HCAdV to mediate apoptosis induction, inhibition of tumor cell growth, and increase of tumor cell death.

\section{Results}

\subsection{HPV Oncogene Specific CRISPR Expressed from HCAdVs Efficiently Disrupts PV E6 Oncogenes}

A schematic overview of the HCAdV vector genomes used in this study is presented in Figure 1. As a control virus, we used the E1- and E3-deleted first generation adenoviral vector $\Delta \mathrm{E} 1-\Delta \mathrm{E} 3-\mathrm{AdV} 5$, which can replicate in HPV-positive cancer cells. Furthermore, two HCAdVs-encoding CRISPR/Cas9 and gRNA against the HPV16 oncogene E6 (HCAdV-CRISPR-HPV16E6gRNA) and the HPV18 oncogene E6 (HCAdV-GFP-CRISPR-HPV18E6gRNA) were generated (Figure 1).

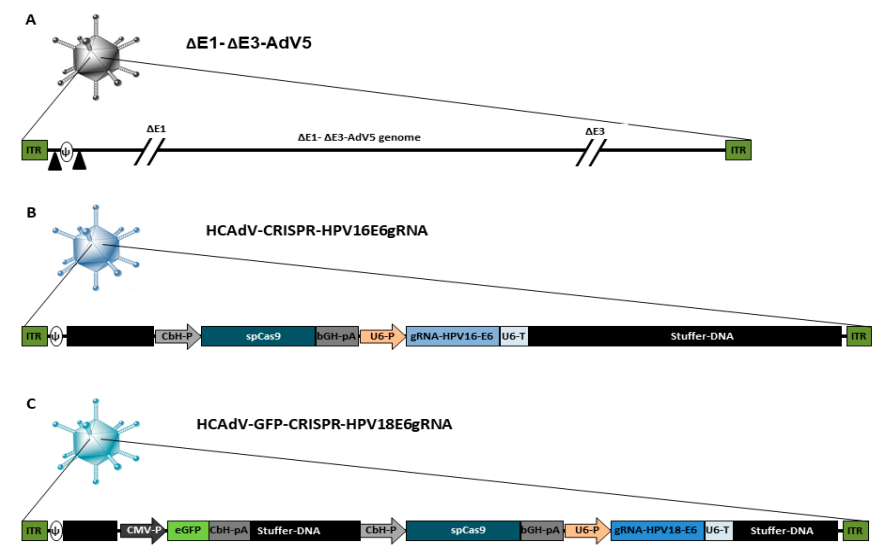

Figure 1. Schematic presentation of the genome organization of the vectors used in this study. (A) $\triangle \mathrm{E} 1-\triangle \mathrm{E} 3-\mathrm{AdV} 5$ contains a wild type AdV5 genome lacking the E1- and E3 gene, the packaging signal is flanked by loxP sites indicated by black triangles. (B) HCAdV-CRISPR-HPV16E6gRNA contains a spCas9-gene from Staphylococcus pyogenes controlled by a $\mathrm{CbH}$-promoter and a bGH-pA and a gRNA expression cassette with specificity toward HPV16-E6 controlled by a human U6-promoter and a U6-terminator sequence. (C) HCAdV-CRISPR-HPV18E6gRNA contains a spCas9-gene controlled by a $\mathrm{CbH}$-promoter and a bGH-pA and a gRNA expression cassette with specificity towards HPV18-E6 controlled by a human U6-Promoter and a U6-terminator sequence. It also contains an enhanced GFP transgene expression cassette controlled by a CMV promoter and a $\mathrm{CbH}-\mathrm{pA}$. CbH-P, chicken $\beta$ actin hybrid promoter; bGH-pA, bovine growth hormone polyadenylation signal; gRNA, guide RNA; U6-P, human U6- small nuclear RNA promoter; U6-pA, U6- small nuclear RNA polyadenylation signal; CMV-P, Cytomegalovirus promoter, $\mathrm{CbH}-\mathrm{pA}$ chicken $\beta$ actin hybrid polyadenylation signal; ITR, inverted terminal repeat; $\Psi$, AdV5 packaging signal. 
To prove CRISPR-mediated mutation induction at the target sites of the respective gRNAs, HeLa and SiHa cells were transduced with HPV18-E6 specific CRISPR-HCAdV or HPV16-E6 specific CRISPR-HCAdV, respectively. Then, $48 \mathrm{~h}$ post-transduction, genomic DNA of HPV18-E6-specific CRISPR-HCAdV-treated HeLa cells and HPV16-E6-specific CRISPR-HCAdV-treated SiHa cells was isolated for mutation detection. HPV-E6 loci surrounding the respective gRNA target sites were amplified by PCR. Resulting PCR amplicons were then subjected to hetero-duplex formation and digested with T7 endonuclease 1. Specific cleavage products of the expected size were detected for HPV18-E6 and HPV16-E6 respectively indicating successful mutation induction at the predicted target sites (Figure 2).
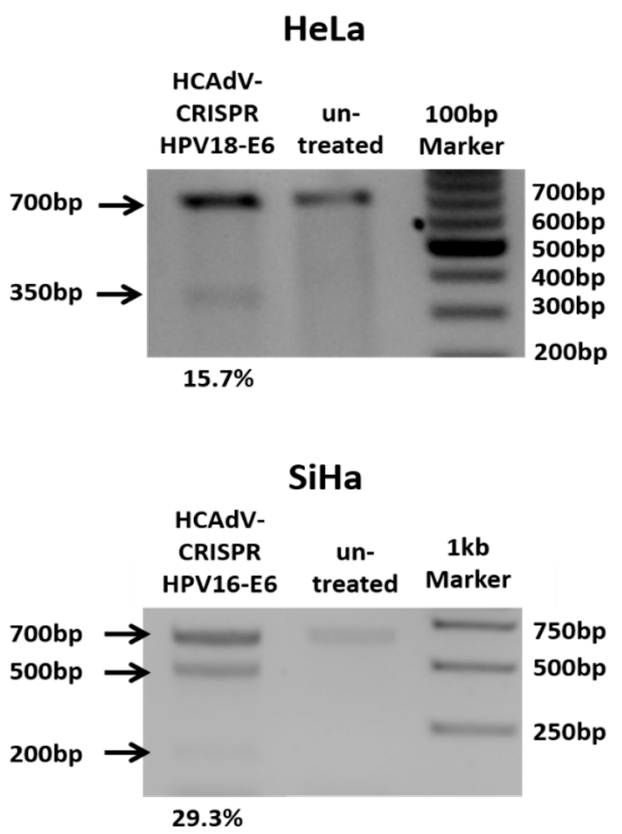

Figure 2. Heteroduplex based mutation detection of HPV16-E6 and HPV18-E6 after treatment of SiHa or HeLa cells at multiplicity of infection (MOI) 500 per cell. $48 \mathrm{~h}$ post transduction the T7E1-assay showed efficient mutagenesis of HPV-E6-genes of cells treated with HPV specific CRISPR HCAdV compared to cells that were not transduced. Arrows indicate the expected size of HPV-E6 specific PCR-products ( $700 \mathrm{bp}$ ) and expected size of mutation specific cleavage products ( $350 \mathrm{bp}$ for HPV18E6 and $200 \mathrm{bp}$ and $500 \mathrm{bp}$ for HPV16E6). Mutation rates calculated in percentages from the differences in band strength of the original PCR product and cleavage products are depicted below.

\subsection{Decrease of HPV Tumor Cell Survival}

To examine the potential of HCAdV armed with HPV-E6 specific CRISPR/Cas9 to reduce HPV tumor specific cell survival, we transduced HeLa, SiHa and CaSki cervical cancer cells as well as HPV negative A549 lung carcinoma cells with HPV18-E6 specific CRISPR-HCAdV, HPV16-E6 specific CRISPR-HCAdV, $\triangle \mathrm{E} 1-\triangle \mathrm{E} 3-\mathrm{AdV} 5$, or AdV storage buffer. Compared to untreated controls or AdV storage buffer treated controls, cervical carcinoma cells treated with HPV-E6-specific CRISPR-HCAdV showed a reduced number of surviving cells (Figure 3). SiHa cells transduced with HPV16-E6-specific CRISPR-HCAdV showed a significant reduction of $85.4 \%$ of surviving cells, whereas transduction with $\triangle \mathrm{E} 1-\triangle \mathrm{E} 3-\mathrm{AdV} 5$ led to a reduction of $44.3 \%$ of viable cells. CaSki cells transduced with HPV16-E6-specific CRISPR-HCAdV showed a reduction of $29.6 \%$ of metabolizing cells (Figure 3 ). In contrast, transduction with $\triangle \mathrm{E} 1-\triangle \mathrm{E} 3-\mathrm{AdV} 5$ resulted in a reduction of $30.4 \%$ of viable cells. In HeLa cells transduced with HPV18-E6-specific CRISPR-HCAdV showed a viability reduction of $33.7 \%$, whereas transduction with $\Delta \mathrm{E} 1-\Delta \mathrm{E} 3-\mathrm{AdV} 5$ decrease viable cells of $15.8 \%$ (Figure 3). Even though a trend for reduction of cell viability can be observed CaSki and HeLa cells, values obtained for these cells were not statistically 
significant. For HPV-negative A549 cells, transduced with CRISPR expressing HCAdV, cell viability was also reduced. Transduction with HPV16-E6 specific CRISPR-HCAdV led to a reduction of 22\% of viable cells and for HPV18-E6 specific CRISPR-HCAdV to a reduction of 52.1\% of viable cells. $\Delta \mathrm{E} 1-\triangle \mathrm{E} 3-\mathrm{AdV} 5$ resulted in a reduction of $36 \%$ when measuring viable cells in A549 cells. Compared with untreated or buffer treated controls, values were not statistically significant (Figure 3).

$\mathrm{SiHa}$

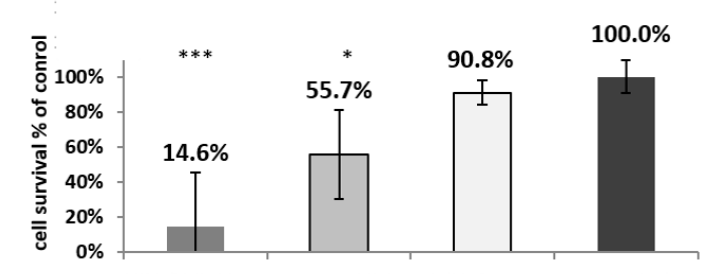

CaSki

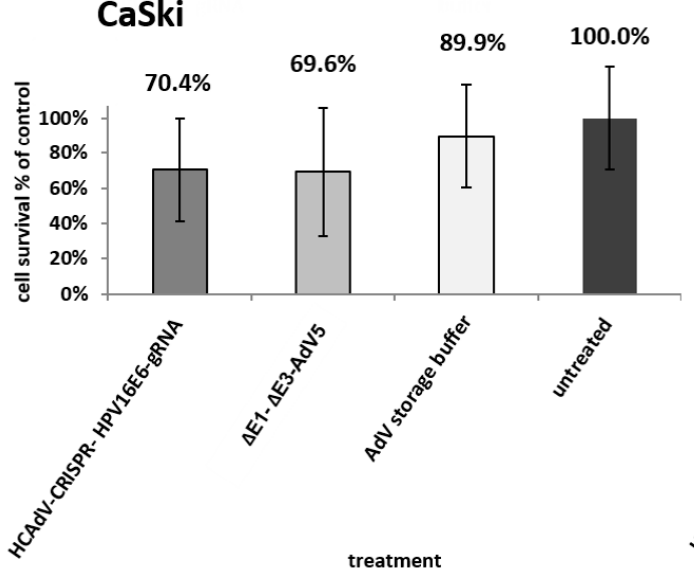

HeLa

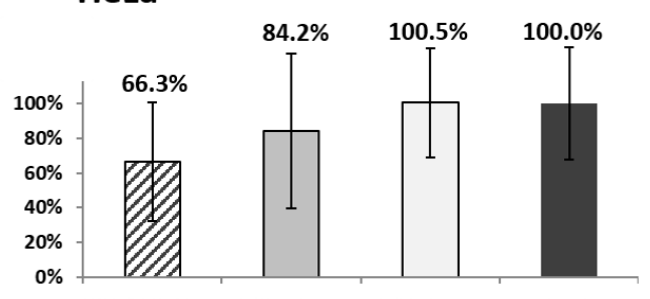

A549

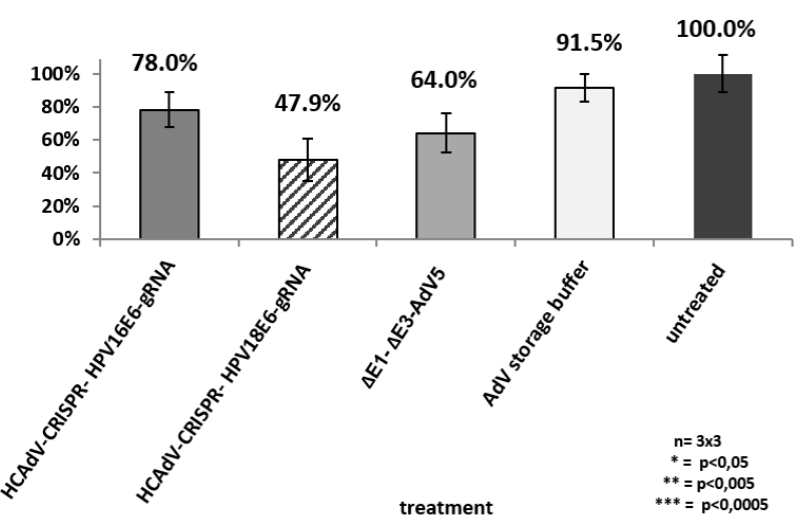

Figure 3. Monitoring of cell survival in SiHa, HeLa, CaSki, and A549 cells. Cells were transduced at confluency with HPV-E6-specific CRISPR/Cas9-HCAdV or $\triangle \mathrm{E} 1-\Delta \mathrm{E} 3-\mathrm{AdV} 5$ with 1000 infectious vector particles per cell. Cell viability was measured using a CCK-8 assay five days post-transduction. HPV-positive tumor cells showed reduced viability compared to untreated controls. Standard deviations of mean values are shown as error bars. Statistically significant differences compared to untreated controls are shown as one, two, or three stars indicating $p$ values $<0.05,<0.005$, and $<0.0005$ respectively.

Following the CCK-8 cell viability screening, the medium was removed, and cells were subjected to methylene blue staining to confirm the previous results using a different methodology that visualizes the healthy attached cells. The results of the methylene blue staining support the results obtained for the CCK-8-based viability assay and showed even stronger effects on the attachment of cells as quantified by the CCK-8 assay. In HeLa, SiHa, and CaSki, a clear decrease of attached cells could be seen after transduction with the respective vector at MOI 1000, whereas untreated controls (MOI 0) or AdV storage-buffer-treated controls were well attached (Supplementary Materials Figure S1). A549 cells showed reduction in cell attachment when treated with HPV18-E6 or HPV16-E6-specific CRISPR-HCAdV or $\triangle \mathrm{E} 1-\Delta \mathrm{E} 3-\mathrm{AdV} 5$ (Figure S1).

\subsection{Cervical Cancer Cell Lines Show Different Susceptibility to AdV5}

To find out whether the differences in the effect of the HPVE6 specific CRISPR/Cas9 expressing HCAdV on different cervical cancer cell lines is caused by different transduction efficiencies of the vector, we determined the susceptibility of $\mathrm{SiHa}, \mathrm{HeLa}$, and CaSki cells to AdV5. We infected each respective cell line with defined numbers of viral particles of a GFP-luciferase expressing E3 deleted AdV5.

$24 \mathrm{~h}$ post transduction with 20 viral particles per cell, quantification of luciferase activity of transduced cells showed a significant 100.4-fold increase in luminescence in SiHa cells compared to CaSki cells, whereas HeLa cells revealed a 2.1-fold increase in luciferase expression levels compared to 
CaSki cells (Figure 4A). At low virus concentration, SiHa cells seem to be more susceptible to AdV5 infection than HeLa and CaSki cells (Figure 4A).

Due to saturation of the luminescence signal at higher viral particle numbers, we compared susceptibility of the different cell lines to AdV5 by quantifying the fluorescent signal from vector-derived GFP expression. Quantification of the mean fluorescence intensity $48 \mathrm{~h}$ post transduction of each respective cell line with 1000 viral particles per cell showed a significant 1.5-fold increased fluorescence signal in SiHa and HeLa cells if directly compared to CaSki cells, respectively. No difference was observed between SiHa and HeLa cells (Figure 4B).
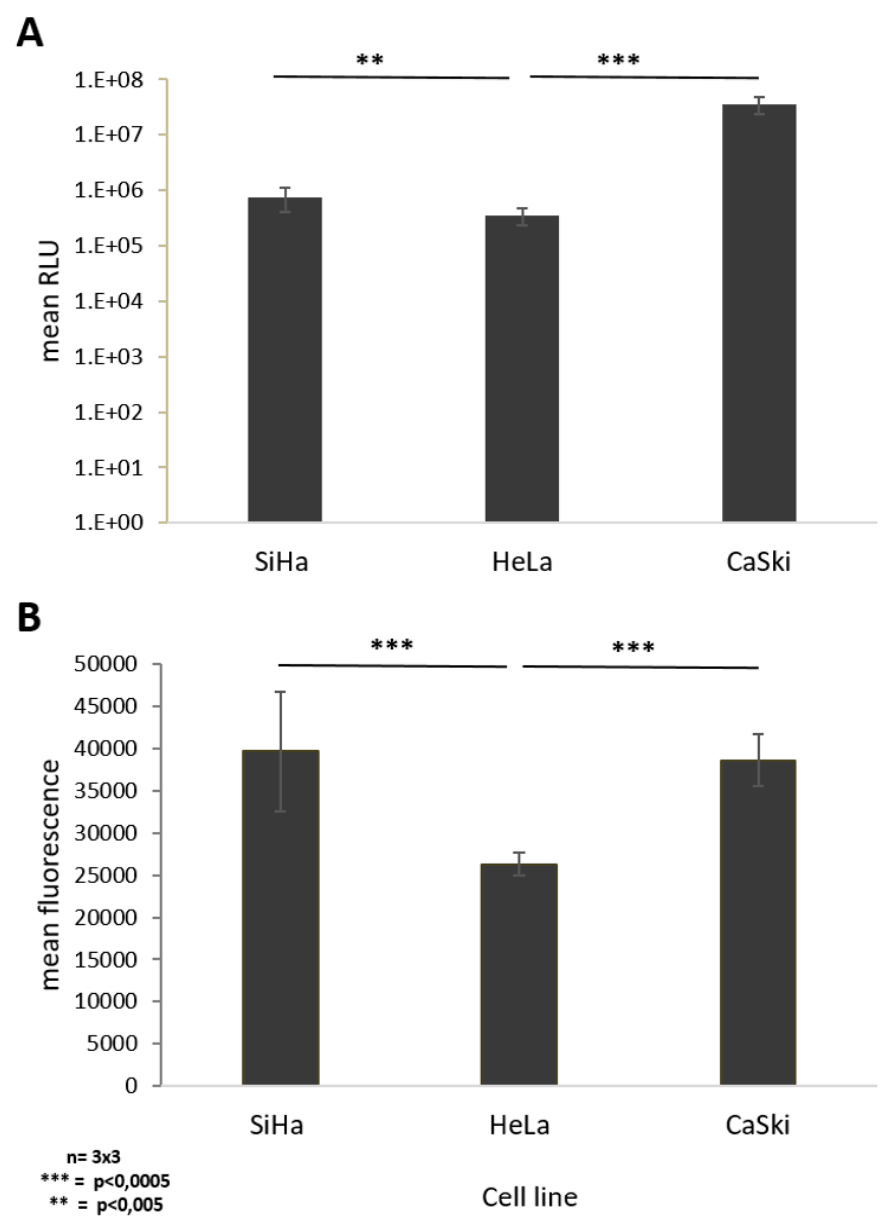

Figure 4. Monitoring cell susceptibility of SiHa, HeLa, and CaSki cells to AdV5. Siha, HeLa, and Caski cells were infected with E3-deleted AdV5-expressing GFP and luciferase at different doses. (A) AdV5 mediated luminescence $24 \mathrm{~h}$ post transduction with 20 viral particles per cell (vpc). (B) AdV 5 mediated fluorescence $48 \mathrm{~h}$ post transduction with $1000 \mathrm{vpc}$. Standard deviations of mean values are shown as error bars. The line above the columns indicate which sampled were compared to each other Statistically significant differences of the cell lines compared to each other are shown as two or three stars, indicating $p$ values $<0.005$, and 0.0005 respectively.

\subsection{Reduction of Proliferation of HPV Positive Cancer Cell Lines}

To investigate whether HPV-E6 specific CRISPR-HCAdV can reduce proliferation of HPV-induced cervical cancer cells, we transduced HPV18 containing HeLa cells, HPV16-positive SiHa and CaSki and $\mathrm{SiHa}$ cervical cancer cells as well as HPV-negative A459 lung carcinoma cells. We applied the vectors HPV18-E6 specific CRISPR-HCAdV, HPV16-E6 specific CRISPR-HCAdV or $\triangle \mathrm{E} 1-\Delta \mathrm{E} 3-\mathrm{AdV} 5$ at MOI 1000 and monitored the increase of viable cells for eight days. Transduction with HPV16-E6-specific CRISPR-HCAdV inhibited cell proliferation of SiHa cells and the number of viable cells significantly 
differed from untreated controls already three days post-transduction. In contrast, transduction with $\Delta \mathrm{E} 1-\Delta \mathrm{E} 3-\mathrm{AdV} 5$ only led to a significant reduction of cell proliferation that was significantly different from untreated controls after day 6 (Figure 5). Transduction with HPV16-E6 specific CRISPR-HCAdV inhibited cell proliferation of CaSki cells and the number of viable cells was significantly reduced compared to untreated controls already four days post-transduction. Transduction with $\Delta \mathrm{E} 1-\Delta \mathrm{E} 3-\mathrm{AdV} 5$ also resulted in a significant reduction of cell proliferation that was significantly different from untreated controls after day 6 (Figure 5). Transduction with HPV18-E6 specific CRISPR-HCAdV strongly inhibited cell proliferation of HeLa cells, which was in sharp contrast to untreated controls already three days post-transduction. Transduction with $\Delta \mathrm{E} 1-\Delta \mathrm{E} 3-\mathrm{AdV} 5$ resulted in a less pronounced reduction of cell proliferation that was still significantly different from untreated controls between days 4-6 (Figure 5).
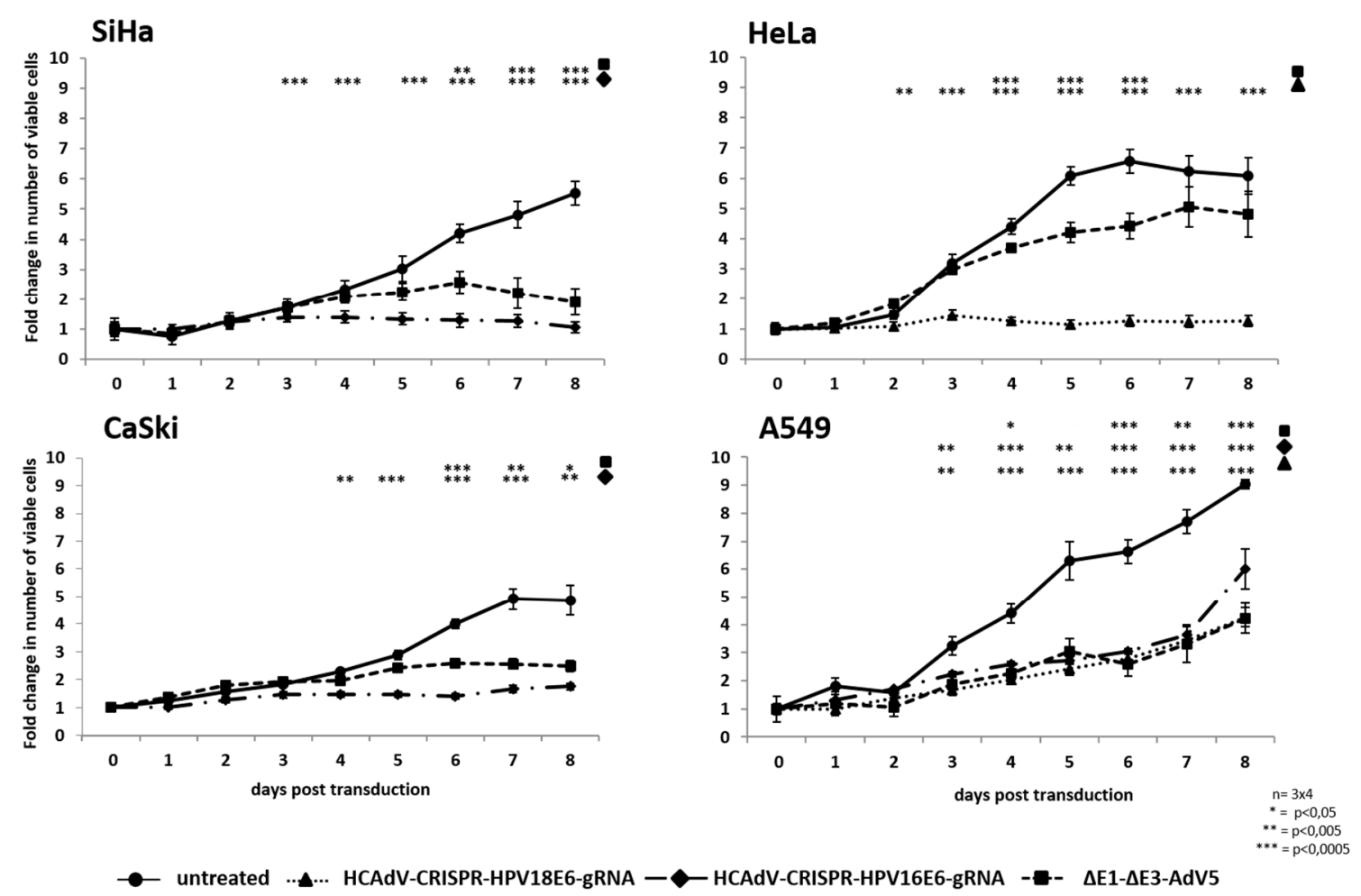

Figure 5. Monitoring of cell proliferation in SiHa, HeLa, CaSki, and A549 cells. Following transduction with the respective HPV-E6-specific CRISPR/Cas9-HCAdV or $\Delta \mathrm{E} 1-\Delta \mathrm{E} 3-\mathrm{AdV} 5$ with 1000 infectious vector particles per cell, cell proliferation was monitored for eight days. The relative number of viable cells at each time point post-transduction was measured by CCK-8 assay. Standard deviations of mean values are depicted as error bars. Statistically significant differences compared to untreated controls are shown as one, two or three stars, indicating $p$ values $<0.05,<0.005$, and $<0.0005$ respectively.

Note that transduction with HPV16-E6-specific CRISPR-HCAdV, HPV18-E6-specific CRISPR-HCAdV, or $\triangle \mathrm{E} 1-\triangle \mathrm{E} 3-\mathrm{AdV} 5$ also reduced cell proliferation of A549 cells, which was different from untreated controls. Even though cell proliferation was reduced proliferation continued until the end of the experiment independent of the treatment (Figure 5). Compared to untreated control cells, treatment with HPV-E6 specific CRISPR-HCAdV or $\triangle \mathrm{E} 1-\Delta \mathrm{E} 3-\mathrm{AdV} 5$ led to a decreased cell proliferation in HPV-induced tumor cells. In HeLa cells, cell proliferation was strongly inhibited by the HPV18-E6-specific CRISPR-HCAdV and $\triangle$ E1- $\triangle$ E3-AdV5. In SiHa and CaSki cells, HPV16-E6-specific CRISPR-HCAdV and HPV18-E6-specific CRISPR-HCAdV and $\Delta$ E1- $\triangle$ E3-AdV5 reduced cell proliferation when compared to untreated cells. In contrast, HPV negative A549 cells were almost unaffected by HPV-E6-specific CRISPR-HCAdV when compared to untreated controls. As seen in HPV-positive cells $\triangle \mathrm{E} 1-\Delta \mathrm{E} 3-\mathrm{AdV} 5$ was also able to inhibit cell proliferation in 
HPV-negative A549 cells. Taken together, these results suggested that tumor cell proliferation inhibition of specific HPV-E6-specific CRISPR-HCAdV is specific for HPV-positive cervical cancer cells and that $\Delta \mathrm{E} 1-\triangle \mathrm{E} 3-\mathrm{AdV} 5$ acts through a different mechanism possibly conditional replication in tumor cells.

\subsection{Vector Treatment Lead to Increase in $p 53$ Protein Levels}

To prove that HCAdV delivery of HPV16E6 or HPV18-specific CRISPR/Cas9 and subsequent E6 mutagenesis increased apoptosis in a p53 dependent manner, we performed cell western analysis to quantify the change in cellular p53 level upon vector transduction. The results show a significant of 1.6-fold and 1.3-fold increase of p53 for SiHa and CaSki cells, respectively, when treated with HCAdV-CRISPR-HPV16E6gRNA. HeLa and A549 cell treated with HCAdV-CRISPR-HPV18E6gRNA showed no change in p53 levels (Figure 6).

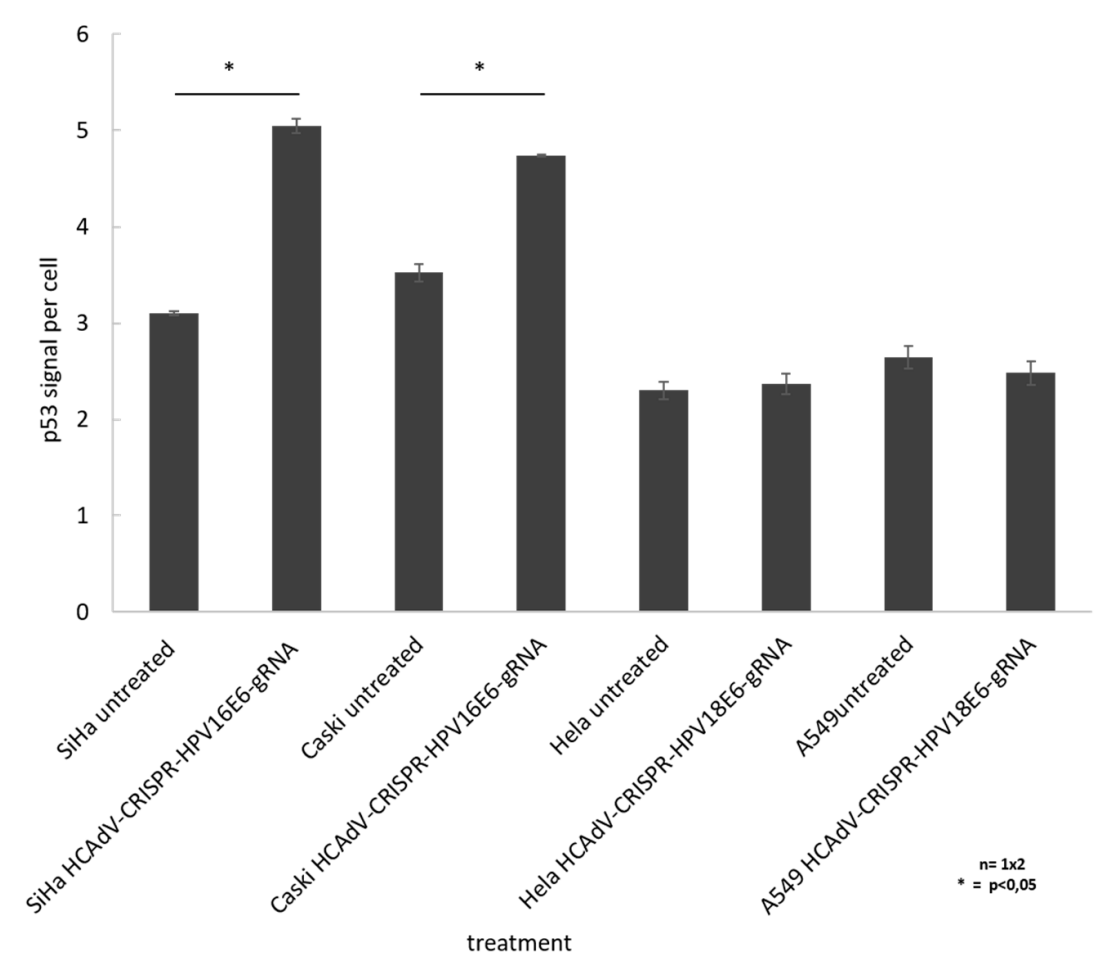

Figure 6. Monitoring change in p53 levels in treated SiHa, HeLa, and CaSki cells. In cell western analyses based on p53 quantification of cells treated with MOI 1000 of respective vectors compared to untreated controls $96 \mathrm{~h}$ post transduction. For each well, the fluorescent intensity values of the p53 antibody measurement at $800 \mathrm{~nm}$ was divided by the mean fluorescent intensity of cell tag700 at $700 \mathrm{~nm}$ to normalize for the cell number (p53 signal per cell). Standard deviations of mean values are shown as error bars. Statistically significant differences compared to untreated controls are shown as one star, indicating $p$ values $<0.05$.

\subsection{Increase in Apoptosis Induction}

As high-risk HPV-E6 proteins interact with the cellular p53 tumor suppressor and mediate its proteasomal degradation, HPV-induced cervical cancer cells can circumvent p53 mediated apoptosis induction. Inactivation of HPV-E6 should inhibit HPV-E6-mediated p53 degradation, leading to a re-accumulation p53 protein and a reactivation of p53 mediated apoptosis induction. To investigate whether HCAdV armed with HPV-E6-specific CRISPR/Cas9 specifically induce cell death in HPV tumor cells by mediating apoptosis induction, we transduced HPV18-containing HeLa cells, SiHa and CaSki cervical cancer cells, and A459 lung carcinoma cells. We applied the vectors HPV18-E6-specific CRISPR-HCAdV, HPV16-E6-specific CRISPR-HCAdV, and $\triangle E 1-\triangle E 3-A d V 5$. Two days post-transduction, the increase of apoptosis induction was determined by measuring 
the activation of effector Caspases 3/7. Compared to untreated controls, transduction of SiHa cells with HPV16-E6 specific CRISPR-HCAdV significantly increased Caspase 3/7 induction (7.1-fold), whereas transduction with $\Delta \mathrm{E} 1-\triangle \mathrm{E} 3-\mathrm{AdV} 5$ only increased Caspase 3/7 induction 1.4-fold (Figure 7). Transduction of CaSki cells with HPV16-E6-specific CRISPR-HCAdV significantly increased Caspase $3 / 7$ induction 2.19-fold and transduction with $\Delta \mathrm{E} 1-\Delta \mathrm{E} 3-\mathrm{AdV} 5$ increased Caspase 3/7 induction 1.28-fold (Figure 7). Transduction of HeLa cells with HPV18-E6 specific CRISPR-HCAdV increased Caspase 3/7 induction 1.49-fold, whereas transduction with $\Delta \mathrm{E} 1-\Delta \mathrm{E} 3-\mathrm{AdV} 5$ significantly increased Caspase $3 / 7$ induction 1.78-fold (Figure 7). Transduction of A549 cells with HPV16-E6-specific CRISPR-HCAdV, HPV18-E6-specific CRISPR-HCAdV, or $\triangle E 1-\triangle E 3-A d V 5$ moderately reduced Caspase 3/7 induction but without any statistical significance.
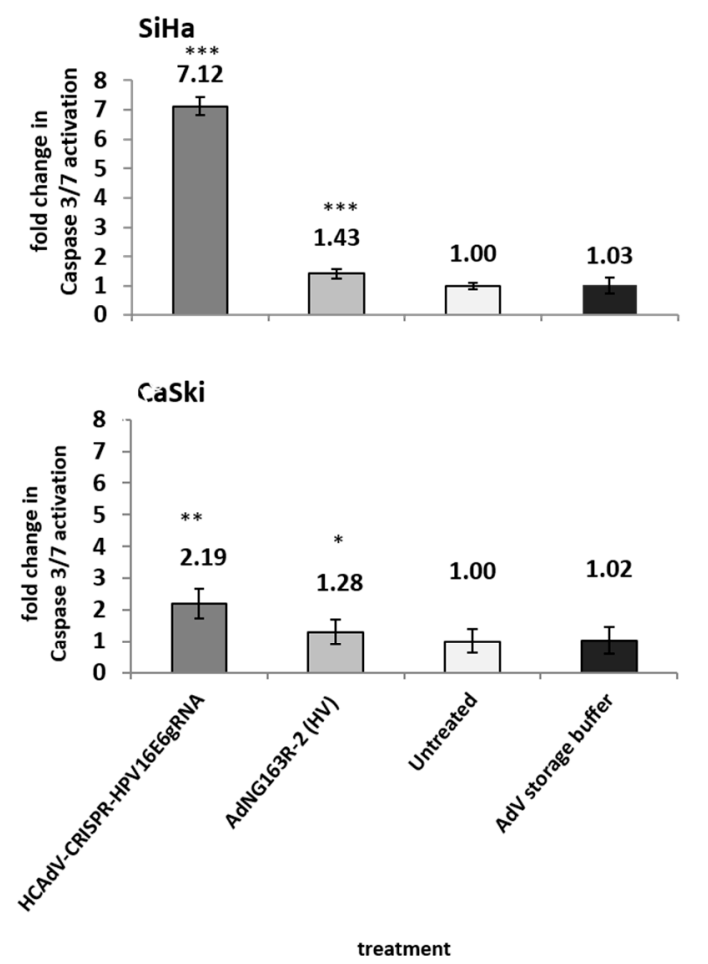

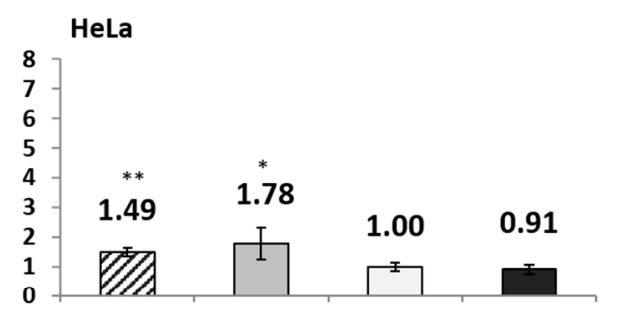

$\$ 549$

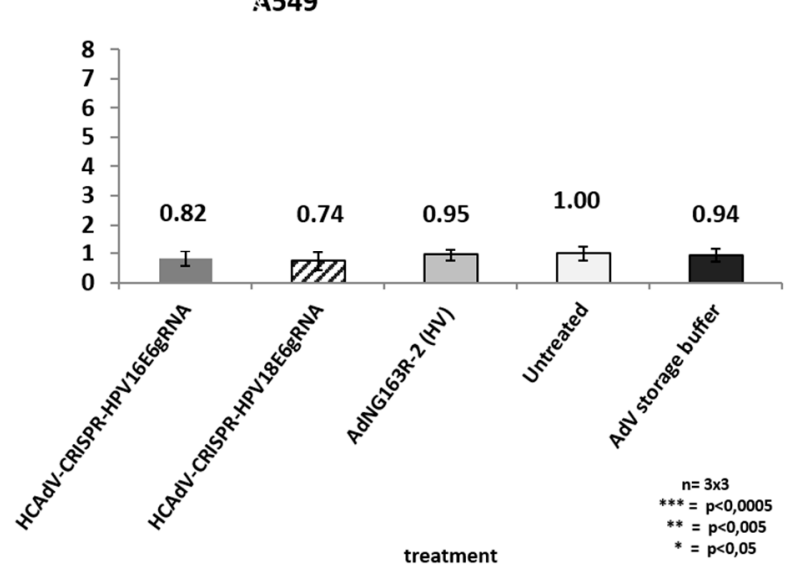

Figure 7. Monitoring apoptosis induction in SiHa, HeLa, CaSki, and A549 cells. Here, $48 \mathrm{~h}$ post-transduction with the respective HPV-E6-specific CRISPR/Cas9-HCAdV and $\triangle \mathrm{E} 1-\Delta \mathrm{E} 3-\mathrm{AdV} 5$ at 1000 infectious vector particles per cell, caspase 3/7 activation was measured. Standard deviations of mean values are shown as error bars. Statistically significant differences compared to untreated controls are shown as one, two, or three stars, indicating $p$ values of $<0.05,<0.005$, and $<0.0005$ respectively.

\section{Discussion}

Previous studies demonstrated that designer-nuclease-mediated destruction of HPV oncogenes are efficient strategies to combat HPV-induced tumors by inducing apoptosis [26-30,41]. To exploit this strategy as a therapeutic intervention, we aimed at translating the findings based on CRISPR/Cas9-mediated HPV oncogene destruction toward a potential in vivo delivery strategy using an all-in-one CRISPR-HCAdVs containing a Cas9 expression cassette and a HPV E6 specific gRNA expression. We tested the effects of the HPVE6 specific CRISPR/Cas9 expressing HCAdV on viability, proliferation and apoptosis induction of cervical cancer cell lines compared to a $\Delta E 1-\Delta E 3-A d V 5-C R A d V$ that kills tumor cells but not non-transformed cells due to the cytopathic effect caused by AdV replication [42-47]. The results of this study prove that HCAdV can be used as delivery vehicles for the HPV-E6-specific CRISPR/Cas9 machinery. Transduction of three different HPV-induced cervical 
cancer cell lines HeLa, CaSki, and SiHa with HPV-oncogene-specific CRISPR-HCAdV inhibited tumor cell proliferation and increased cell death though induction of apoptosis.

Noteworthy, even though the same cell numbers were infected with the same MOI, the antitumor effects in the cell lines investigated in this study differ. In SiHa cells, the antitumor effects of our vector treatment were strong, whereas CaSki cells showed no or at least weak response to the treatment (Figures 3 and 5-7). The response to vector treatment in part reflects the different susceptibility of the studied cell lines to the vector. When treated with low viral particle numbers, SiHa cells are 100-fold more susceptible to AdV5 than CaSki cells (Figure 4A). In contrast, at high viral particle numbers per cell, the difference in vector susceptibility is less pronounced. HeLa and SiHa cells are 1.5-fold more susceptible than CaSki cells with no differences between SiHa and CaSki cells (Figure 4B). Therefore, differences in vector susceptibility only partially explain the differences in response to vector treatment.

Another difference between the cervical cancer cells studied here is the genome copy number of HPV genomes integrated into the chromosomes. Those differences might also contribute to the differences in response to vector treatment. SiHa cells contain 1-2 HPV16 genome copies integrated into their chromosomes, whereas CaSki cells contain more than 600 copies of HPV16 in 11 chromosomal sites, as well as low copy numbers of partial HPV18 genomes [48-51]. The intermediate response in HeLa cells to the vector treatment might also be explained by the intermediate number of HPV18 genomes [52] in HeLa cells. HeLa cells contain at least five copies of the HPV18 genome [53,54]. Therefore, the difference in response to the vector treatment could be explained by a large number of uncut HPV copies remaining within HeLa and even more in CaSki cells leading to ongoing anti-apoptotic E6 effects within these cells. In SiHa cells, CRISPR/Cas9 seemed to have mutated enough E6 sequences on genome level to inhibit E6 effects. HPV copy numbers in cervical tumors depends on the integration frequencies and chromosome duplication events and can vary between different lesions. As HPV integration status and copy number seem to influence the effect of an anti-HPV-CRISPR approach, molecular diagnostics determining the HPV type, integration status, and viral load, can be predictive for the efficiency of such an anti-HPV-CRISPR HCAdV treatment. As we observed differences in the susceptibility of the different cells to the vector and differences in response to the treatment, pretreatment diagnostics could also help predict the optimal vector dose. This strategy enables to identify the optimal dose to achieve the desired response in a cell line-dependent manner and in the future to develop a personalized therapy approach for patients.

It remains unclear why the cell survival of HPV negative A549 cells was affected after transduction with HPV oncogene-specific CRISPR-HCAdVs. All used vectors including the $\Delta$ E1- $\Delta$ E3-AdV5 non-CRISPR control influenced cell viability in A549 cells. This is probably due to the high transduction efficiencies of adenoviral vectors in A549 cells. It is well established that A549 cells are highly susceptible to adenovirus infection. As all vectors negatively influenced A549 cell survival and proliferation this is probably not due to the genetic cargo, but rather a general reaction to the viral transduction. Zhen et al. [26,29], as well as Kennedy et al. [28], who published the gRNAs used in this study, did not report any off-target effects after plasmid transfection. As cellular toxicity due to Cas9 overexpression has been reported in hematopoietic stem cells [55], the presence of Cas9 protein could have led to decreased cell viability of A549 cells. Before further developing anti-HPV-CRISPR HCAdV treatment, further studies including other HPV-negative control cell lines and especially control vectors containing a non-specific gRNA or vectors containing no gRNA are needed to elucidate whether decreased viability in HPV-negative cells is due to the vector treatment, the presence of Cas9 and potential unspecific action, or an artifact resulting from the assay used to quantify viable cells.

Compared to the CRISPR-expressing HCAdVs, $\triangle \mathrm{E} 1-\triangle \mathrm{E} 3-\mathrm{AdV} 5$ showed slightly stronger inhibitory effects on cell viability. However, HPV-specific CRISPR/Cas9-expressing HCAdV led to much stronger Caspase 3/7 induction in HPV-positive cells. However, in A549, no significant induction of apoptosis was observed, indicating that the inhibitory effects on viability and proliferation of HPV positive cells were specific to the CRISPR/Cas9-mediated E6 disruption and induction of apoptosis. Previous studies have shown that transfection of HPV-positive cervical cancer cells with HPV-specific CRISPR/Cas9 
expression plasmids lead to increased p53 levels [26,30]. Following adenoviral delivery of HPV specific CRISPR/Cas9 we also observed an increase in p53 levels (Figure 6), indicating that the elimination of $\mathrm{HPV}$ oncogenes leads to a re-accumulation or less p53 degradation, which explains the higher number of cells that showed increased Caspase 3/7 activity upon vector treatment (Figure 7).

HCAdV armed with HPV-E6-specific CRISPR/Cas9 drive cell death and inhibition of cell proliferation of HPV-positive cervical cancer cells through apoptosis induction, whereas $\triangle E 1-\triangle E 3-A d V 5$ did not significantly increase apoptosis induction in HPV-positive or HPV-negative cell lines. The observed $\Delta \mathrm{E} 1-\Delta \mathrm{E} 3$-AdV5-mediated tumor cell killing is apoptosis independent and could rather be explained as a result of viral replication. This shows that the effects seen after transduction with HPV-specific CRISPR/Cas9-expressing HCAdV are related to the specific effects of it CRISPR/Cas9 cargo rather than the transduction process or potential replication of remaining helper virus particles within the HCAdV preparations. The versatility of the CRISPR/Cas9 system in combination with HCAdV delivery which can be constructed in a facilitated way through our CRISPR-HCAdV production pipeline [38] offers the possibility to develop personalized treatment depending on the causative HPV type present in a respective patient tumor. Here, precise diagnostics of the HPV type and viral load within the tumor will help to choose the optimal treatment regimen.

Translation of the present results towards preclinical in vivo models is challenging. Xenograft models in immune-deficient mice or transgenic mice expressing HPV oncogenes are a considerable model system that can be exploited. Here, the route of administration will be of particular interest. Systemic injection of AdV vectors based on AdV serotype 5 will efficiently transduce the liver [56], limiting the effectiveness for treating epithelial tumors. Regarding the necessity to treat epithelia with precursor lesions, invasive tumors and infected areas without transformation in close proximity [5], alternative strategies should be considered. As AdVs are able to penetrate the upper layers of epithelium of the mouse skin without inducing cytotoxic effects [57], vector delivery through microporation [58,59] could be a relevant option.

Besides the route of administration modification of the vectors used for this approach can contribute to optimize delivery to relevant cells. Chemical modification of vector capsids [60] or using Darpins as adaptors between target cells and vector [61] could direct the vectors to the desired cell type to increase specificity and efficiency of the approach. Recently, an alternative AdV serotype became available [62-64]. Vectorization of such serotypes could contribute to an improved efficiency of the approach.

However, the setup of transgenes used in the HCAdV-expressing HPV-specific CRISPR/Cas9 used in this study does not exploit the full antitumor potential of what HCAdV could achieve. Additional gRNAs targeting could be included to enhance the anti-HPV effects. Addition of RNA interference directed against HPV oncogene mRNA $[14,18]$ could be added to reach synergistic effects. A combined expression of immune modulators [65] or anti-neoangiogenic factors [66] could also be considered. Arming CRAdV, with HPV oncogene specific CRISPR/Cas9 could enhance efficacy through synergistic effects. Therefore, combining the effects of HPV-oncogene-specific designer nucleases with oncolytic virotherapy is also a reasonable strategy that deserves further commitment.

\section{Materials and Methods}

\subsection{Viral Vectors}

An E1and E3-deleted, first-generation Adenovirus AdNG163R-2 ( $\triangle \mathrm{E} 1-\triangle \mathrm{E} 3-\mathrm{AdV} 5)$ was produced as previously described [37]. HPV16 and HPV18-specific CRISPR/Cas9-expressing HCAdV based on human AdV serotype 5 (AdV5) were produced as previously described [38]. In brief, HCAdV-CRISPR-HPV16E6gRNA and HCAdV-CRISPR-HPV18E6gRNA contained a staphylococcus pyogenes Cas9 gene driven by a $\mathrm{CbH}$ promoter and a gRNA expression unit controlled by human U6-RNA promoter. HCAdV-CRISPR-HPV18E6gRNA contained an additional GFP expression cassette driven by CMV promoter (Figure 2). The gRNA sequences for HPV18-E6 
(GCGCTTTGAGGATCCAACA) and HPV16-E6 (CAACAGTTACTGCGACGTG) were previously published [26,29].

Quantification of infectious virus particles within the vector preparations was carried out as previously described $[67,68]$. Briefly, HEK293 cells were transduced with defined volumes of the purified vectors. Three hours post transduction cells were washed with $1 \mathrm{x}$ phospahate-buffered saline (PBS) prior to harvesting and harvested cells were centrifuged and resuspended in 1x PBS to remove non-infective particles and centrifuged again. Following isolation of genomic DNA from transduced cells, HCAdV vector genome copy numbers were determined by HCAdV-specific q-PCR.

\subsection{Cell Lines and Cell Culture}

The cervical cancer cell lines SiHa and CaSki containing HPV16 and HeLa-containing HPV18 genomes integrated into their chromosomal DNA as well as HPV-negative A549 lung carcinoma cells were cultured in DMEM medium supplemented with $10 \%$ fetal bovine serum (FBS, PAN Biotec, Aidenbach, Germany), $100 \mathrm{U} / \mathrm{mL}$ Penicillin and $100 \mu \mathrm{g} / \mathrm{mL}$ Streptomycin (PAN Biotec) unless stated otherwise. Cells were grown at $37^{\circ} \mathrm{C}$ and $5 \% \mathrm{CO}_{2}$.

\subsection{Mutation Induction and Detection}

Determination of genome targeting efficiency using T7 Endonuclease I was performed using a protocol adapted from previously published protocols [69]. Briefly, $2 \times 10^{6}$ cells were seeded into 24-well plates and subsequently infected with the respective HPV type-specific CRISPR-HCAdVs. Then, $48 \mathrm{~h}$ post-infection, genomic DNA was extracted from the cells for subsequent PCR using a Nucleo Spin Tissue kit (Macherey-Nagel, Düren, Deutschland). PCR amplicons covering the respective HPV-E6 region surrounding the respective gRNA binding sites was amplified using Phusion high fidelity DNA polymerase kit (New England Biolabs, Frankfurt, Germany) according to manufacturer's instructions. The HVP18-E6 amplicon was generated using the primer-set HPV18T7E1fwd (5' CTTGCATAACTATATCCACTCCC $3^{\prime}$ ) and HPV18E6rev (5' ATTCAACGGTTTCTGGCAC 3') yielding a PCR product of $656 \mathrm{bp}$ and HPV16E 6 region was amplified using the primer-set HPV16E6fwd (5' TGAACCGAAACCGGTTAGTA 3') and HPV16E6rev (5' TGAACCGAAACCGGTTAGTA 3') (30) yielding a PCR product of $660 \mathrm{bp}$. PCR products were purified by ethanol precipitation by filling the PCR volume up to $100 \mu \mathrm{L}$ with $\mathrm{H}_{2} \mathrm{O}$, adding of $10 \mu \mathrm{L} \mathrm{NaAc}(3 \mathrm{M}, \mathrm{pH} 8.0)$ and $250 \mu \mathrm{L}$ ice cold $\mathrm{EtOH}(100 \%)$ followed by centrifugation for $10 \mathrm{~min}$, high speed. The DNA-pellet was washed with $500 \mu \mathrm{L} \mathrm{EtOH}(70 \%)$ and centrifuged for $5 \mathrm{~min}$ at high speed. After centrifugation, the supernatant was discarded, and the pellet was air dried and resuspended in $17.5 \mu \mathrm{L} \mathrm{H}_{2} \mathrm{O}$. Purified amplicons were supplemented with $2 \mu \mathrm{L}$ of Buffer NEB2 (New England Biolabs, Ipswich, MA, USA) and subjected to heteroduplex formation by heating them to $95^{\circ} \mathrm{C}$ and ramping down to room temperature using a cooling rate of $0.1{ }^{\circ} \mathrm{C} / \mathrm{s}$. After heteroduplex formation, $0.5 \mu \mathrm{L}$ of T7E1 enzyme (New England Biolabs) was added and the mixture was incubated for $15 \mathrm{~min}$ at $37^{\circ} \mathrm{C}$. The reaction was stopped by adding purple loading dye (New England Biolabs, containing $10 \mathrm{mM}$ EDTA, $0.08 \%$ SDS) and separated on a $2 \%$ agarose gel and at $\sim 90 \mathrm{~V}$ for $>45 \mathrm{~min}$. Mutation rate was calculated according to the formula: $\%$ gene modification $=100 \times(1-(1-$ fraction cleaved $) 1 / 2)[69]$.

\subsection{Cell Viability Assay and Crystal Violet Staining}

We seeded $1.5 \times 10^{6} \mathrm{HeLa}$, CaSki, SiHa, and A549 cells into 24-well plates. Five hours later, cells were transduced with $0,0.1,1.0,10,100$, or 1000 infectious particles of the respective HPV16 or HPV18-specific CRISPR/Cas9-expressing HCAdV or AdNG163R-2. To rule out potential cytotoxic effects of the solvent used to dilute the vectors, negative controls cells were treated with serial dilution of AdV storage buffer ( $50 \mathrm{mM}$ Hepes $\mathrm{pH} 7.4,100 \mathrm{mM} \mathrm{NaCl}, 10 \%$ Glycerol), equaling the highest volume of buffer that was applied for MOI 1000 of the HPV16-specific CRISPR/Cas9-expressing HCAdV. Five days post-transduction, cell-viability was determined using the Cell Counting Kit-8 (CCK-8; Sigma, St. Louis, MO, USA) following manufacturer's instructions. Briefly, $10 \mu \mathrm{L}$ of CCK8 substrate 
were applied to each well containing $100 \mu \mathrm{L}$ of cell culture medium and incubated for $1 \mathrm{~h}$ to allow viable cells to convert the CCK-8 substrate into a colored dye. Adsorption at $450 \mathrm{~nm}$ was measured using a GENios multi-plate reader (TECAN, Crailsheim, Germany). After CCK-8 readout, the medium was removed by three consecutive washing steps with $1 \times$ PBS and fixed in $10 \%$ formaldehyde in PBS for $10 \mathrm{~min}$ at room temperature, followed by three consecutive washing steps with $1 \times$ PBS. Adherent cells were stained with crystal violet staining solution for $10 \mathrm{~min}$. Excess staining solution was removed by gently washing the plates in tap water. Subsequently plates were air dried and kept for photo documentation. All experiments were performed three times with triplicates for each sample.

\subsection{Determination of Cellular Susceptibility to Human AdV Serotype 5 (AdV5)}

To determine the susceptibility of the cell lines used in this study by means of GFP reporter gene expression, 40,000 cells of HeLa, SiHa, or CaSki cells were seeded in a 96-well plate. Then, $5 \mathrm{~h}$ post-seeding, cells were infected with 1000 viral particles of an E3 deleted AdV5-expressing GFP and luciferase that was produced as previously described [62]. Forty-eight hours post-transduction, virus-mediated GFP fluorescence within transduced cells was quantified using an Infinity 200 pro multi-plate reader (TECAN). To determine the susceptibility of the cell lines used in this study by means of luciferase reporter gene expression, 40,000 cells of $\mathrm{HeLa}$, SiHa, or CaSki cells were seeded per well in a 96-well plate. Five hours post-seeding, cells were infected with 20 viral particles of an E3 deleted AdV5 expressing GFP and luciferase. Then, $24 \mathrm{~h}$ post-transduction, quantification of virus-mediated luciferase activity of transduced cells was carried out using the dual-luciferase reporter assay (Promega, Walldorf, Germany) following the manufacturer's instructions. Luminescence measurement was performed using an Infinity 200 pro multi-plate reader (TECAN). Experiments were repeated three times in triplicates.

\subsection{Proliferation Assay}

We seeded $1 \times 10^{3} \mathrm{HeLa}$, CaSki, SiHa or A549 cells per well into 96-well plates, respectively, and transduced with 1000 infectious vector particles per cell of the HPV16 or HPV18 specific CRISPR/Cas9 expressing HCAdV or AdNG163R-2, respectively. The number of viable cells for each treatment was determined using the Cell Counting Kit-8 (CCK-8; Sigma, St. Louis, MO, USA) in $24 \mathrm{~h}$ intervals in quadruplicates for seven days. All experiments were performed three times with quadruplicates for each sample.

\subsection{In Cell Western Analysis of Cellular p53}

To analyze changes in cellular p53 protein levels in response to HPV-specific CRISPR/Cas9-expressing HCAdV treatment, we performed in cell western analysis. Here, 25,000 cells of the respective cell line were seeded to each well of a 96 well plate. SiHa and CaSki cells were treated with HAdV-CRISPR-HPV16E6gRNA and Hela, and A549 cells were treated with HCAdV-CRISPR-HPV18E6gRNA at MOI of 1000, respectively. Controls were left untreated. Then, $96 \mathrm{~h}$ post-transduction, the medium was removed, and cells were fixed using $1 \times$ PBS, $4 \%$ paraformaldehyde for $20 \mathrm{~min}$ at room temperature. Fixed cells were washed three times using $1 \times$ PBS, $0.1 \%$ triton X100 for five minutes to permeabilize the cells. Following cell permeabilization, wells were incubated with blocking solution (LI-COR, Lincoln, NE, USA) for $90 \mathrm{~min}$ at room temperature. After blocking, wells were incubated with AlexaFluor790 coupled anti-p53 antibody (p53 (Do-1): sc-126; Santa Cruz, Dallas, Germany) and CellTag 700 Stain (LI-COR, Lincoln, NE, USA) diluted 1:50 and 1:500, respectively, in blocking solution for $150 \mathrm{~min}$ at room temperature. Background controls only received anti p53 antibody or cell tag 700, respectively. Finally, plates were washed with 1 x PBS, 0,1\% tween 20 for five times at room temperature before plates were air dryed and the 700 and $800 \mathrm{~nm}$ fluorescent signals of all wells were quantified using the Odyssey CLx scanner (LI-COR) and image studio 5.2 software (LI-COR). Measurements were carried out at a resolution of $169 \mu \mathrm{m}$ using a focus depth of $3 \mathrm{~mm}$. For each well, the fluorescent intensity values of the p53 antibody $(800 \mathrm{~nm})$ was divided by the mean 
fluorescent intensity of cell tag700 $(700 \mathrm{~nm})$ to normalize for the cell number. Data were analyzed in duplicates.

\subsection{Apoptosis Assay}

We seeded $2.5 \times 10^{4} \mathrm{HeLa}$, CaSki, SiHa, or A549 cells into black-walled 96-well plates with a transparent bottom. Between experimental groups, one row was left empty to prevent the luminescent signal to influence neighboring wells. Five hours post-seeding, cells were transduced with HPV16 or HPV18-specific CRISPR/Cas9-expressing HCAdV or the E1-deleted, first generation Adenovirus (AdNG163R-2), respectively. To rule out potential apoptotic effects of the solvent used to dilute the vectors, untransfected cells were treated with AdV storage buffer (50 mM HEPES, pH 7.4, $100 \mathrm{mM}$ $\mathrm{NaCl}, 10 \%$ Glycerol), equaling the highest volume of virus that was applied for MOI 1000 of the HPV16-specific CRISPR/Cas9-expressing HCAdV. Untreated cells were incubated with cell culture medium alone. Then, $48 \mathrm{~h}$ post-transduction, apoptosis induction was monitored by measuring caspase 3/7 activation using luminescent caspase 3/7 glow assay (Promega) following the manufacturer's instructions. Luciferase signal indicating Caspase 3/7-mediated substrate cleavage was detected using a GENios multi-plate reader (TECAN). Cell culture medium alone or medium supplemented with the AdV storage buffer was measured on the same 96-well plate to determine background signal. These background values were subtracted from the sample values. All experiments were performed three times with quadruplicates for each sample.

\subsection{Statistical Analysis}

Statistical significance of differences between experimental groups and untreated controls were analyzed using Students t-test using the software GraphPad Prism 8 (San Diego, CA, USA). Significant differences with $p$ values of $p<0.0005, p<0.005$, or $p<0.05$ are depicted as ${ }^{* * *}$, ${ }^{* *}$ or * respectively.

\section{Conclusions}

In summary, this study shows a proof-of-concept for using the CRISPR/Cas9 technology delivered by the most advanced adenoviral vector (HCAdV) to treat HPV-derived tumors. We conclude that HCAdV can serve as HPV-specific cancer gene therapeutic agents when armed with HPV-type-specific CRISPR/Cas9. We believe that our approach can contribute to treatment options specific for the respective HPV type present in each individual tumor. The next step is the translation of this approach into relevant in vivo animal models and, in the long-term run, this approach may lead to a novel concept in personalized tumor therapy.

Supplementary Materials: The following are available online at http://www.mdpi.com/2072-6694/12/7/1934/s1, Figure S1. Oncolysis assay to monitor cell viability.

Author Contributions: Conceptualization, E.E.-S.; Methodology and experiments, E.E.-S., S.H., L.S. and M.S.; E.E.-S.; Data Analysis, S.H.; Writing—original draft preparation, E.E.-S. and A.E.; Writing-review and editing, E.E.-S. and A.E.; Project administration, E.E.-S. and A.E.; funding acquisition, E.E.-S. and A.E. All authors have read and agreed to the published version of the manuscript.

Funding: This research was funded by the foundation "Stiftung Tumorforschung Kopf-Hals."

Acknowledgments: We thank the research group of B.A. for sharing CaSki and SiHa cells and their helpful advice.

Conflicts of Interest: The authors declare no conflict of interest. The funders had no role in the design of the study; in the collection, analyses, or interpretation of data; in the writing of the manuscript, or in the decision to publish the results.

\section{References}

1. Zur Hausen, H. Papillomaviruses and cancer: From basic studies to clinical application. Nat. Rev. Cancer 2002, 2, 342-350. [CrossRef] 
2. Munoz, N.; Bosch, F.X.; de Sanjose, S.; Herrero, R.; Castellsague, X.; Shah, K.V.; Snijders, J.F.; Meijer, C.J.L.M.L. Epidemiologic classification of human papillomavirus types associated with cervical cancer. N. Engl. J. Med. 2003, 348, 518-527. [CrossRef] [PubMed]

3. Hubbers, C.U.; Akgul, B. HPV and cancer of the oral cavity. Virulence 2015, 6, 244-248. [CrossRef]

4. Nindl, I.; Gottschling, M.; Stockfleth, E. Human papillomaviruses and non-melanoma skin cancer: Basic virology and clinical manifestations. Dis. Markers 2007, 23, 247-259. [CrossRef] [PubMed]

5. Stockfleth, E. The importance of treating the field in actinic keratosis. J. Eur. Acad Dermatol. Venereol. 2017, 31 (Suppl. 2), 8-11. [CrossRef] [PubMed]

6. Lechner, M.S.; Laimins, L.A. Inhibition of p53 DNA binding by human papillomavirus E6 proteins. J. Virol. 1994, 68, 4262-4273. [CrossRef]

7. Munger, K.; Werness, B.A.; Dyson, N.; Phelps, W.C.; Harlow, E.; Howley, P.M. Complex formation of human papillomavirus E7 proteins with the retinoblastoma tumor suppressor gene product. EMBO J. 1989, 8, 4099-4105. [CrossRef]

8. Dyson, N.; Howley, P.M.; Munger, K.; Harlow, E. The human papilloma virus-16 E7 oncoprotein is able to bind to the retinoblastoma gene product. Science 1989, 243, 934-937. [CrossRef]

9. Amici, C.; Visintin, M.; Verachi, F.; Paolini, F.; Percario, Z.; Di Bonito, P.; Mandarino, A.; Affabris, E.; Venuti, A.; Accardi, L. A novel intracellular antibody against the E6 oncoprotein impairs growth of human papillomavirus 16-positive tumor cells in mouse models. Oncotarget 2016, 7, 15539-15553. [CrossRef]

10. Guo, C.P.; Liu, K.W.; Luo, H.B.; Chen, H.B.; Zheng, Y.; Sun, S.N.; Zhang, Q.; Huang, L. Potent anti-tumor effect generated by a novel human papillomavirus (HPV) antagonist peptide reactivating the $\mathrm{pRb} / \mathrm{E} 2 \mathrm{~F}$ pathway. PLoS ONE 2011, 6, e17734. [CrossRef]

11. Butz, K.; Denk, C.; Ullmann, A.; Scheffner, M.; Hoppe-Seyler, F. Induction of apoptosis in human papillomaviruspositive cancer cells by peptide aptamers targeting the viral E6 oncoprotein. Proc. Natl. Acad. Sci. USA 2000, 97, 6693-6697. [CrossRef] [PubMed]

12. Liu, Y.; Liu, Z.; Androphy, E.; Chen, J.; Baleja, J.D. Design and characterization of helical peptides that inhibit the E6 protein of papillomavirus. Biochemistry 2004, 43, 7421-7431. [CrossRef] [PubMed]

13. Zheng, Y.F.; Rao, Z.G.; Zhang, J.R. Effects of anti-HPV16 E6-ribozyme on the proliferation and apoptosis of human cervical cancer cell line CaSKi. Di Yi Jun Yi Da Xue Xue Bao 2002, 22, 496-498. [PubMed]

14. Butz, K.; Ristriani, T.; Hengstermann, A.; Denk, C.; Scheffner, M.; Hoppe-Seyler, F. siRNA targeting of the viral E6 oncogene efficiently kills human papillomavirus-positive cancer cells. Oncogene 2003, 22, 5938-5945. [CrossRef]

15. Jiang, M.; Milner, J. Selective silencing of viral gene expression in HPV-Positive human cervical carcinoma cells treated with siRNA, a primer of RNA interference. Oncogene 2002, 21, 6041-6048. [CrossRef] [PubMed]

16. Yamato, K.; Yamada, T.; Kizaki, M.; Ui-Tei, K.; Natori, Y.; Fujino, M.; Nishihara, T.; Ikeda, Y.; Nasu, Y.; Saigo, K.; et al. New highly potent and specific E6 and E7 siRNAs for treatment of HPV16 positive cervical cancer. Cancer Gene Ther. 2008, 15, 140-153. [CrossRef] [PubMed]

17. Nishida, H.; Matsumoto, Y.; Kawana, K.; Christie, R.J.; Naito, M.; Kim, B.S.; Miyata, K.; Taguchi, A.; Tomio, K.; Yamashita, A.; et al. Systemic delivery of siRNA by actively targeted polyion complex micelles for silencing the E6 and E7 human papillomavirus oncogenes. J. Control. Release 2016, 231, 29-37. [CrossRef] [PubMed]

18. Sato, N.; Saga, Y.; Uchibori, R.; Tsukahara, T.; Urabe, M.; Kume, A.; Fujiwara, H.; Suzuki, M.; Ozawa, K.; Mizukami, H.; et al. Eradication of cervical cancer in vivo by an AAV vector that encodes shRNA targeting human papillomavirus type 16 E6/E7. Int. J. Oncol. 2018. [CrossRef]

19. Niu, X.Y.; Peng, Z.L.; Duan, W.Q.; Wang, H.; Wang, P. Inhibition of HPV 16 E6 oncogene expression by RNA interference in vitro and in vivo. Int. J. Gynecol. Cancer 2006, 16, 743-751. [CrossRef]

20. Chang, J.T.C.; Kuo, T.F.; Chen, Y.J.; Chiu, C.C.; Lu, Y.C.; Li, H.F.; Shen, C.-R.; Cheng, A.-J. Highly potent and specific siRNAs against E6 or E7 genes of HPV16- or HPV18-infected cervical cancers. Cancer Gene Ther. 2010, 17, 827. [CrossRef]

21. Lea, J.S.; Sunaga, N.; Sato, M.; Kalahasti, G.; Miller, D.S.; Minna, J.D.; Muller, C.Y. Silencing of HPV 18 oncoproteins With RNA interference causes growth inhibition of cervical cancer cells. Reprod. Sci. 2007, 14, 20-28. [CrossRef] [PubMed]

22. Schiwon, M.; Ehrke-Schulz, E.; Oswald, A.; Bergmann, T.; Michler, T.; Protzer, U.; Ehrhardt, A. One-Vector System for Multiplexed CRISPR/Cas9 against Hepatitis B Virus cccDNA Utilizing High-Capacity Adenoviral Vectors. Mol. Ther.-Nucl. Acids 2018, 12, 242-253. [CrossRef] [PubMed] 
23. Weber, N.D.; Stone, D.; Jerome, K.R. TALENs targeting HBV: Designer endonuclease therapies for viral infections. Mol. Ther. J. Am. Soc. Gene Ther. 2013, 21, 1819-1820. [CrossRef] [PubMed]

24. Bloom, K.; Ely, A.; Mussolino, C.; Cathomen, T.; Arbuthnot, P. Inactivation of hepatitis B virus replication in cultured cells and in vivo with engineered transcription activator-like effector nucleases. Mol. Ther. J. Am. Soc. Gene Ther. 2013, 21, 1889-1897. [CrossRef]

25. Mino, T.; Mori, T.; Aoyama, Y.; Sera, T. Gene- and protein-delivered zinc finger-staphylococcal nuclease hybrid for inhibition of DNA replication of human papillomavirus. PLoS ONE 2013, 8, e56633. [CrossRef]

26. Kennedy, E.M.; Kornepati, A.V.; Goldstein, M.; Bogerd, H.P.; Poling, B.C.; Whisnant, A.W.; Kastan, M.B.; Cullen, B.R. Inactivation of the human papillomavirus E6 or E7 gene in cervical carcinoma cells by using a bacterial CRISPR/Cas RNA-guided endonuclease. J. Virol. 2014, 88, 11965-11972. [CrossRef]

27. Hu, Z.; Yu, L.; Zhu, D.; Ding, W.; Wang, X.; Zhang, C.; Wang, L.; Jiang, X.; Shen, H.; He, D.; et al. Disruption of HPV16-E7 by CRISPR/Cas system induces apoptosis and growth inhibition in HPV16 positive human cervical cancer cells. Biomed. Res. Int. 2014, 2014, 612823. [CrossRef]

28. Liu, Y.C.; Cai, Z.M.; Zhang, X.J. Reprogrammed CRISPR-Cas9 targeting the conserved regions of HPV6/11 E7 genes inhibits proliferation and induces apoptosis in E7-transformed keratinocytes. Asian J. Androl. 2016, 18, 475-479. [CrossRef]

29. Zhen, S.; Hua, L.; Takahashi, Y.; Narita, S.; Liu, Y.H.; Li, Y. In vitro and in vivo growth suppression of human papillomavirus 16-positive cervical cancer cells by CRISPR/Cas9. Bioch. Biophys. Res. Commun. 2014, 450, 1422-1426. [CrossRef]

30. Yu, L.; Wang, X.; Zhu, D.; Ding, W.; Wang, L.; Zhang, C.; Jiang, X.; Shen, H.; Liao, S.; Ma, D.; et al. Disruption of human papillomavirus $16 \mathrm{E} 6$ gene by clustered regularly interspaced short palindromic repeat/Cas system in human cervical cancer cells. Onco Targets Ther. 2015, 8, 37-44. [CrossRef]

31. Jubair, L.; Fallaha, S.; McMillan, N.A.J. Systemic Delivery of CRISPR/Cas9 Targeting HPV Oncogenes Is Effective at Eliminating Established Tumors. Mol. Ther. J. Am. Soc. Gene Ther. 2019. [CrossRef] [PubMed]

32. Heise, C.; Kirn, D.H. Replication-Selective adenoviruses as oncolytic agents. J. Clin. Investig. 2000, 105, 847-851. [CrossRef] [PubMed]

33. Russell, S.J.; Peng, K.W.; Bell, J.C. Oncolytic virotherapy. Nat. Biotechnol. 2012, 30, 658-670. [CrossRef] [PubMed]

34. Yuan, M.; Webb, E.; Lemoine, N.R.; Wang, Y. CRISPR-Cas9 as a Powerful Tool for Efficient Creation of Oncolytic Viruses. Viruses 2016, 8, 72. [CrossRef]

35. Jager, L.; Ehrhardt, A. Persistence of high-capacity adenoviral vectors as replication-defective monomeric genomes in vitro and in murine liver. Hum. Gene Ther. 2009, 20, 883-896. [CrossRef]

36. Parks, R.J.; Chen, L.; Anton, M.; Sankar, U.; Rudnicki, M.A.; Graham, F.L. A helper-dependent adenovirus vector system: Removal of helper virus by Cre-Mediated excision of the viral packaging signal. Proc. Natl. Acad. Sci. USA 1996, 93, 13565-13570. [CrossRef]

37. Palmer, D.; Ng, P. Improved system for helper-dependent adenoviral vector production. Mol. Ther. J. Am. Soc. Gene Ther. 2003, 8, 846-852. [CrossRef] [PubMed]

38. Ehrke-Schulz, E.; Schiwon, M.; Leitner, T.; David, S.; Bergmann, T.; Liu, J.; Ehrhardt, A. CRISPR/Cas9 delivery with one single adenoviral vector devoid of all viral genes. Sci. Rep. 2017, 7, 17113. [CrossRef] [PubMed]

39. Schiedner, G.; Morral, N.; Parks, R.J.; Wu, Y.; Koopmans, S.C.; Langston, C.; Graham, F.L.; Beaudet, A.L.; Kochanek, S. Genomic DNA transfer with a high-capacity adenovirus vector results in improved in vivo gene expression and decreased toxicity. Nat. Genet. 1998, 18, 180-183. [CrossRef]

40. Ehrhardt, A.; Kay, M.A. A new adenoviral helper-dependent vector results in long-term therapeutic levels of human coagulation factor IX at low doses in vivo. Blood 2002, 99, 3923-3930. [CrossRef] [PubMed]

41. Yoshiba, T.; Saga, Y.; Urabe, M.; Uchibori, R.; Matsubara, S.; Fujiwara, H.; Mizukami, H. CRISPR/Cas9-mediated cervical cancer treatment targeting human papillomavirus E6. Oncol. Lett. 2019, 17, 2197-2206. [CrossRef] [PubMed]

42. Chellappan, S.; Kraus, V.B.; Kroger, B.; Munger, K.; Howley, P.M.; Phelps, W.C.; Nevins, J.R. Adenovirus E1A, simian virus 40 tumor antigen, and human papillomavirus E7 protein share the capacity to disrupt the interaction between transcription factor E2F and the retinoblastoma gene product. Proc. Natl. Acad. Sci. USA 1992, 89, 4549-4553. [CrossRef] [PubMed] 
43. Sang, N.; Avantaggiati, M.L.; Giordano, A. Roles of p300, pocket proteins, and hTBP in E1A-mediated transcriptional regulation and inhibition of p53 transactivation activity. J. Cell. Biochem. 1997, 66, 277-285. [CrossRef]

44. Whyte, P.; Williamson, N.M.; Harlow, E. Cellular targets for transformation by the adenovirus E1A proteins. Cell 1989, 56, 67-75. [CrossRef]

45. Steinwaerder, D.S.; Carlson, C.A.; Lieber, A. DNA Replication of First-Generation Adenovirus Vectors in Tumor Cells. Hum. Gene Ther. 2000, 11, 1933-1948. [CrossRef]

46. Steinwaerder, D.S.; Carlson, C.A.; Lieber, A. Human papilloma virus E6 and E7 proteins support DNA replication of adenoviruses deleted for the E1A and E1B genes. Mol. Ther. 2001, 4, 211-216. [CrossRef]

47. Abou, E.L.; Hassan, M.A.; van der Meulen-Muileman, I.; Abbas, S.; Kruyt, F.A. Conditionally replicating adenoviruses kill tumor cells via a basic apoptotic machinery-independent mechanism that resembles necrosis-like programmed cell death. J. Virol. 2004, 78, 12243-12251. [CrossRef]

48. Raybould, R.; Fiander, A.; Wilkinson, G.W.; Hibbitts, S. HPV integration detection in CaSki and SiHa using detection of integrated papillomavirus sequences and restriction-site PCR. J. Virolog. Methods 2014, 206, 51-54. [CrossRef]

49. Yee, C.; Krishnan-Hewlett, I.; Baker, C.C.; Schlegel, R.; Howley, P.M. Presence and expression of human papillomavirus sequences in human cervical carcinoma cell lines. Am. J. Pathol. 1985, 119, 361-366.

50. Baker, C.C.; Phelps, W.C.; Lindgren, V.; Braun, M.J.; Gonda, M.A.; Howley, P.M. Structural and transcriptional analysis of human papillomavirus type 16 sequences in cervical carcinoma cell lines. J. Virol. 1987, 61, 962-971. [CrossRef] [PubMed]

51. Mincheva, A.; Gissmann, L.; zur Hausen, H. Chromosomal integration sites of human papillomavirus DNA in three cervical cancer cell lines mapped by in situ hybridization. Med. Microbiol. Immunol. 1987, 176, 245-256. [CrossRef] [PubMed]

52. Maglennon, G.A.; McIntosh, P.; Doorbar, J. Persistence of viral DNA in the epithelial basal layer suggests a model for papillomavirus latency following immune regression. Virology 2011, 414, 153-163. [CrossRef] [PubMed]

53. Adey, A.; Burton, J.N.; Kitzman, J.O.; Hiatt, J.B.; Lewis, A.P.; Martin, B.K.; Qiu, R.; Lee, C.; Shendure, J. The haplotype-resolved genome and epigenome of the aneuploid HeLa cancer cell line. Nature 2013, 500, 207-211. [CrossRef] [PubMed]

54. Macville, M.S.E.; Padilla-Nash,H.; Keck, C.; Ghadimi, B.M.; Zimonjic, D.; Popescu, N.; Ried, T. Comprehensive and definitive molecular cytogenetic characterization of HeLa cells by spectral karyotyping. Cancer Res. 1999, 59, 141-150.

55. Li, C.; Psatha, N.; Gil, S.; Wang, H.; Papayannopoulou, T.; Lieber, A. HDAd5/35(++) Adenovirus Vector Expressing Anti-CRISPR Peptides Decreases CRISPR/Cas9 Toxicity in Human Hematopoietic Stem Cells. Mol. Ther. Methods Clin. Dev. 2018, 9, 390-401. [CrossRef]

56. Alemany, R.; Suzuki, K.; Curiel, D.T. Blood clearance rates of adenovirus type 5 in mice. J. Gen. Virol. 2000, 81 Pt 11, 2605-2609. [CrossRef]

57. Lu, B.; Federoff, H.J.; Wang, Y.; Goldsmith, L.A.; Scott, G. Topical Application of Viral Vectors for Epidermal Gene Transfer. J. Investig. Dermatol. 1997, 108, 803-808. [CrossRef]

58. Bramson, J.; Dayball, K.; Evelegh, C.; Wan, Y.H.; Page, D.; Smith, A. Enabling topical immunization via microporation: A novel method for pain-free and needle-free delivery of adenovirus-based vaccines. Gene Ther. 2003, 10, 251-260. [CrossRef]

59. Weiss, R.; Hessenberger, M.; Kitzmuller, S.; Bach, D.; Weinberger, E.E.; Krautgartner, W.D.; Hauser-Kronberger, C.; Malissen, B.; Boehler, C.; Kalia, Y.N.; et al. Transcutaneous vaccination via laser microporation. J. Control. Release 2012, 162, 391-399. [CrossRef]

60. Yoon, A.R.; Hong, J.; Kim, S.W.; Yun, C.O. Redirecting adenovirus tropism by genetic, chemical, and mechanical modification of the adenovirus surface for cancer gene therapy. Exp. Opin. Drug Deliv. 2016, 13, 843-858. [CrossRef]

61. Dreier, B.; Honegger, A.; Hess, C.; Nagy-Davidescu, G.; Mittl, P.R.; Grutter, M.G.; Belousova, N.; Mikheeva, G.; Krasnykh, V.; Plückthun, A. Development of a generic adenovirus delivery system based on structure-guided design of bispecific trimeric DARPin adapters. Proc. Natl. Acad. Sci. USA 2013, 110, E869-E877. [CrossRef] [PubMed] 
62. Zhang, W.; Fu, J.; Liu, J.; Wang, H.; Schiwon, M.; Janz, S.; Schaffarcyzk, L.; von de Golta, L.; Ehrke-Schulz, E.; Solanki, M.; et al. An engineered Virus Library as a Resource for the Spectrum-Wide Exploration of Virus and Vector Diversity. Cell Rep. 2017, 19, 1698-1709. [CrossRef] [PubMed]

63. Zhang, W.; Fu, J.; Ehrhardt, A. Novel Vector Construction Based on Alternative Adenovirus Types via Homologous Recombination. Hum. Gene Ther. Methods 2018, 29, 124-134. [CrossRef]

64. Zhang, W.; Ehrhardt, A. Getting genetic access to natural adenovirus genomes to explore vector diversity. Virus Genes 2017. [CrossRef]

65. Cerullo, V.; Capasso, C.; Vaha-Koskela, M.; Hemminki, O.; Hemminki, A. Cancer-Targeted Oncolytic Adenoviruses for Modulation of the Immune System. Curr. Cancer Drug Targets 2018, 18, 124-138. [CrossRef] [PubMed]

66. Angarita, F.A.; Acuna, S.A.; Ottolino-Perry, K.; Zerhouni, S.; McCart, J.A. Mounting a strategic offense: Fighting tumor vasculature with oncolytic viruses. Trends Mol. Med. 2013, 19, 378-392. [CrossRef] [PubMed]

67. Ehrke-Schulz, E.; Zhang, W.; Schiwon, M.; Bergmann, T.; Solanki, M.; Liu, J.; Boehme, P.; Leitner, T.; Ehrhardt, A. Cloning and Large-Scale Production of High-Capacity Adenoviral Vectors Based on the Human Adenovirus Type 5. J. Vis Exp. 2016, e52894. [CrossRef]

68. Jager, L.; Hausl, M.A.; Rauschhuber, C.; Wolf, N.M.; Kay, M.A.; Ehrhardt, A. A rapid protocol for construction and production of high-capacity adenoviral vectors. Nat. Protoc. 2009, 4, 547-564. [CrossRef]

69. Guschin, D.Y.; Waite, A.J.; Katibah, G.E.; Miller, J.C.; Holmes, M.C.; Rebar, E.J. A rapid and general assay for monitoring endogenous gene modification. Methods Mol. Biol. 2010, 649, 247-256. [CrossRef]

(C) 2020 by the authors. Licensee MDPI, Basel, Switzerland. This article is an open access article distributed under the terms and conditions of the Creative Commons Attribution (CC BY) license (http://creativecommons.org/licenses/by/4.0/). 